Pacific

Journal of

Mathematics

PROJECTABILITY AND UNIQUENESS OF $F$-STABLE IMMERSIONS WITH PARTIALLY FREE BOUNDARIES

FRANK MÜLLER AND SVEN WINKLMANN 


\title{
PROJECTABILITY AND UNIQUENESS OF $F$-STABLE IMMERSIONS WITH PARTIALLY FREE BOUNDARIES
}

\author{
FRANK MÜLLER AND SVEN WINKLMANN
}

\begin{abstract}
We study immersed critical points $X$ of an elliptic parametric functional $\mathscr{F}(X)=\int_{B} F\left(X_{u} \wedge X_{v}\right) d u d v$ that are spanned into a partially free boundary configuration $\{\Gamma, \mathscr{S}\}$ in $\mathbb{R}^{3}$. We suppose that $\mathscr{S}$ is a cylindrical support surface and that $\Gamma$ is a closed Jordan arc with a simple convex projection. Under geometrically reasonable assumptions on $\{\Gamma, \mathscr{S}\}, F$, and $X$ we prove the projectability and uniqueness of stable immersions. This generalizes a result for minimal surfaces obtained by Hildebrandt and Sauvigny.
\end{abstract}

\section{Introduction}

It is well known that one cannot expect uniqueness for disc-type solutions of Plateau's problem spanning an arbitrary closed Jordan curve $\Gamma \subset \mathbb{R}^{3}$. However, $\Gamma$ bounds exactly one minimal surface if it has a simple projection onto a planar convex curve; this is a celebrated theorem of Radó [1926], with a contribution by Kneser [1926]. Moreover, this surface must in fact be a graph. Sauvigny [1982] was able to generalize this result to surfaces with prescribed mean curvature under an additional stability assumption.

More generally, Hildebrandt and Sauvigny studied the partially free boundary problem for minimal surfaces inside boundary configurations $\{\Gamma, \mathscr{Y}\}$, consisting of a closed Jordan arc $\Gamma$ with a simple convex projection and a cylindrical support surface $\mathscr{S}$. They proved various uniqueness results and the existence of graph representations; see [Hildebrandt and Sauvigny 1991; 1992; 1995]. Again, this result was extended in [Müller 2005] to stable surfaces of prescribed mean curvature.

Here we consider this partially free boundary problem for elliptic parametric functionals of the type

$$
\mathscr{F}(X)=\int_{B} F\left(X_{u} \wedge X_{v}\right) d u d v
$$

MSC2000: 53C42, 35J65, 49Q10.

Keywords: F-minimal immersions, partially free boundaries, uniqueness, projectability, Wulff shape.

Winklmann was financially supported by the Deutsche Forschungsgemeinschaft. 
whose integrand $F: \mathbb{R}^{3} \backslash\{0\} \rightarrow \mathbb{R}^{+}$represents a smooth elliptic Lagrangian satisfying the homogeneity relation

$$
F(t z)=t F(z) \quad \text { for all } z \in \mathbb{R}^{3}, t>0 .
$$

Obviously, $\mathscr{F}$ generalizes the classical area functional

$$
\mathscr{A}(X)=\int_{B}\left|X_{u} \wedge X_{v}\right| d u d v
$$

obtained in the case $F(z)=|z|$.

Using sophisticated tools from the direct methods in the calculus of variations, Hildebrandt and von der Mosel [1999] studied Plateau's problem for general elliptic parametric functionals of the form

$$
\mathscr{F}(X)=\int_{B} F\left(X, X_{u} \wedge X_{v}\right) d u d v
$$

they also addressed the partially free boundary problem [2002]. For a detailed survey on the existence and regularity theory as well as further remarks on the literature, see [Hildebrandt and von der Mosel 2005].

Investigating the functional $\mathscr{F}$ from a more geometric point of view, Winklmann [2003] and Clarenz and von der Mosel [2004] studied immersed critical points, the so-called $F$-stationary immersions, under Plateau-type boundary conditions. This leads to surfaces of vanishing or, more generally, prescribed anisotropic mean curvature, allowing extensions of Radó's and Sauvigny's projectability and uniqueness results.

Here we obtain similar results for immersed surfaces with partially free boundaries. In particular, we extend the uniqueness result of [Hildebrandt and Sauvigny 1995] in an appropriate manner and prove graph representations for stable critical points, or $F$-stable immersions in short, in the cylindrical boundary configuration $\{\Gamma, \mathscr{Y}\}$. (Concerning anisotropic capillary surfaces with free boundaries, see [Koiso and Palmer 2006].)

Specifically, in Section 2 we formulate general assumptions and collect basic facts on $F$-stationary immersions with partially free boundaries; Lemma 2.5 might be of independent interest. In Section 3 we show that the free boundary remains inside $\Sigma \times \mathbb{R}$ (Lemma 3.1) and prove that the surface is transversal to the fixed boundary (Lemma 3.2). We also derive an equation for the surface normal at the free boundary (Lemma 3.3). In Section 4 we compute the second variation of $\mathscr{F}$ (Theorem 4.1) and use the previous results to construct an admissible test function in the stability inequality (Lemma 4.2). In Section 5 we finally prove the projectability of $F$-stable immersions (Theorem 5.1). This leads to the desired 
uniqueness result (Theorem 5.2) via a comparison principle for mixed boundary value problems of minimal surface type.

\section{Notation and preliminary results}

A boundary configuration $\{\Gamma, \mathscr{Y}\}$ consists of a closed Jordan arc $\Gamma \subset \mathbb{R}^{3}$ of class $C^{3}$ with endpoints $P_{1}, P_{2}$ and an embedded support manifold $\mathscr{Y} \subset \mathbb{R}^{3}$ of class $C^{3}$ such that $\mathscr{Y} \cap \Gamma=\left\{P_{1}, P_{2}\right\}$. We also suppose that $\Gamma$ meets $\mathscr{S}$ with a positive angle at these points.

Definition 2.1. A boundary configuration $\{\Gamma, \mathscr{Y}\}$ is named projectable if

(a) $\mathscr{S}=\Sigma_{0} \times \mathbb{R}$ is a cylinder surface over the planar Jordan curve $\Sigma_{0} \in C^{3}$, which decomposes the $x^{1}, x^{2}$-plane $E$ into two unbounded domains.

(b) $\Gamma$ is representable as a graph over $E: \Gamma=\left\{\left(x^{1}, x^{2}, \gamma\left(x^{1}, x^{2}\right)\right):\left(x^{1}, x^{2}\right) \in \underline{\Gamma}\right\}$, where $\gamma\left(x^{1}, x^{2}\right) \in C^{3}(\underline{\Gamma})$ denotes the height function and $\underline{\Gamma} \in C^{3}$ the projection of $\Gamma$ onto $E$.

Let $\Sigma_{0} \in C^{3}$ be parametrized by $\sigma=\sigma(s),-\infty<s<+\infty$, with arc length $\left|\sigma^{\prime}(s)\right| \equiv 1$. Suppose that $P_{k}=\left(\sigma\left(s_{k}\right), \gamma\left(\sigma\left(s_{k}\right)\right), k=1,2\right.$, for $-\infty<s_{1}<s_{2}<+\infty$. If we set $\Sigma:=\left\{\sigma(s): s_{1}<s<s_{2}\right\}$, the closed Jordan curve $\underline{\Gamma} \cup \Sigma$ bounds a simply connected domain $G \subset E$.

Definition 2.2. A projectable boundary configuration $\{\Gamma, \mathscr{Y}\}$ is admissible if

(a) $\Gamma$ is convex with respect to $G$ and does not meet $\Sigma_{0}$ perpendicularly, and

(b) for each $s \in\left(-\infty, s_{1}\right) \cup\left(s_{2},+\infty\right)$, the normal line

$$
L(s):=\left\{p \in E:\left\langle p-\sigma(s), \sigma^{\prime}(s)\right\rangle=0\right\}
$$

meets $\bar{G} \cup \Sigma_{0}$ only at the point $\sigma(s)$.

We also introduce the tangent $t(x):=\left(\sigma^{\prime}(s), 0\right)$ for $x \in\{\sigma(s)\} \times \mathbb{R}, s \in \mathbb{R}$. With the aid of $e_{3}:=(0,0,1)$ we define $n(x):=t(x) \wedge e_{3}$ and $\kappa(x):=-\left\langle\left(\sigma^{\prime \prime}(s), 0\right), n(x)\right\rangle$ for $x \in\{\sigma(s)\} \times \mathbb{R}, s \in \mathbb{R}$. We can assume that $n(x)$ points to the exterior of $G$. Obviously,

$$
t(x), e_{3} \in T_{x} \mathscr{Y}, \quad n(x) \perp T_{x} \mathscr{S} \text { for all } x \in \mathscr{Y},
$$

where $T_{x} \mathscr{Y}$ denotes the tangent space of $\mathscr{Y}$ at $x$.

Let $B:=\left\{w=(u, v) \in \mathbb{R}^{2}: u^{2}+v^{2}<1, v>0\right\}$ denote the semidisc. The boundary $\partial B$ consists of the interval $I:=(-1,1) \times\{0\}$ and the closed semicircle $C:=\partial B \backslash I$. In the sequel, we consider immersions $X: \bar{B} \rightarrow \mathbb{R}^{3}$ of class $C^{0}\left(\bar{B}, \mathbb{R}^{3}\right) \cap$ $C^{3}\left(\bar{B} \backslash\{-1,1\}, \mathbb{R}^{3}\right)$ with their Gauss map

$$
N: \bar{B} \backslash\{-1,1\} \rightarrow \mathbb{R}^{3}, \quad N(w):=\frac{X_{u} \wedge X_{v}}{\left|X_{u} \wedge X_{v}\right|}
$$


possessing finite area

$$
\mathscr{A}(X)=\int_{B} d A<\infty .
$$

Here $d A=\left|X_{u} \wedge X_{v}\right| d u d v$ denotes the surface element with respect to the induced metric $g$. In order to extend the projectability and uniqueness result of [Hildebrandt and Sauvigny 1995] to parametric functionals, we introduce the following class $\mathscr{C}(\Gamma, \mathscr{S})$ of immersions:

Definition 2.3. An immersion $X=X(u, v) \in C^{0}\left(\bar{B}, \mathbb{R}^{3}\right) \cap C^{3}\left(\bar{B} \backslash\{-1,1\}, \mathbb{R}^{3}\right)$ with finite area $\mathscr{A}(X)<\infty$ is called admissible, and we write $X \in \mathscr{C}(\Gamma, \mathscr{Y})$, if

(a) $\left.X\right|_{C}: C \rightarrow \Gamma$ maps $C$ topologically onto $\Gamma$ and $X(-1,0)=P_{1}, X(1,0)=P_{2}$, and

(b) $X(I) \subset \mathscr{Y}$.

Later we will need the following regularity assumptions, which allow us to control the curvature of an $F$-stationary immersion $X \in \mathscr{C}(\Gamma, \mathscr{Y})$ at the corners $w= \pm 1$.

Condition (R). The total curvature of $X$ is bounded, i.e.,

$$
\int_{B}|K| d A<\infty
$$

and the limits

$$
N( \pm 1):=\lim _{w \rightarrow \pm 1} N(w)
$$

exist.

Remark. For stationary minimal surfaces, i.e. the case $F(z)=|z|$, one can show that both conditions (2-1), (2-2) are satisfied. In fact, this follows from asymptotic expansions at the corners $w= \pm 1$, see [Dierkes et al. 1992, Section 8.4]. Thus Condition $(\mathrm{R})$ seems geometrically reasonable.

For $X \in \mathscr{C}(\Gamma, \mathscr{Y})$ we now consider the parametric functional

$$
\mathscr{F}(X)=\int_{B} F(N) d A
$$

with a Lagrangian $F \in C^{3}\left(\mathbb{R}^{3} \backslash\{0\}, \mathbb{R}\right) \cap C^{0}\left(\mathbb{R}^{3}, \mathbb{R}\right)$ satisfying the homogeneity relation (1-1). Throughout this paper, $F$ is assumed to be positive:

$$
F(z)>0 \text { for all } z \neq 0 \text {. }
$$

In addition, we always assume $F$ to be elliptic; that is, the restriction of

$$
F_{z z}(z)=\left(\frac{\partial^{2} F}{\partial z^{\alpha} \partial z^{\beta}}(z)\right)_{\alpha, \beta=1,2,3}
$$


to $z^{\perp}:=\left\{\zeta \in \mathbb{R}^{3}:\langle\zeta, z\rangle=0\right\}$ is a positive-definite linear mapping for all $z \neq 0$.

Geometrically, the ellipticity condition implies that $F$ represents a support function of the convex body

$$
\bigcap_{z \neq 0}\left\{y \in \mathbb{R}^{3}:\langle y, z\rangle \leq F(z)\right\} .
$$

Its boundary $\mathcal{W}_{F}$ gives us the convex surface parametrized by

$$
F_{z}: S^{2} \rightarrow \mathbb{R}^{3}, \quad z \mapsto F_{z}(z) .
$$

In the terminology of [Taylor 1978], $\mathscr{W}_{F}=F_{z}\left(S^{2}\right)$ is called the Wulff shape.

Given $X \in \mathscr{C}(\Gamma, \mathscr{S})$, we say that a smooth family $\bar{X}: \bar{B} \times\left(-\varepsilon_{0}, \varepsilon_{0}\right) \rightarrow \mathbb{R}^{3}$ of immersions is an admissible variation of $X$ if we have $\bar{X}(\cdot, 0)=X$,

$$
Y:=\left.\frac{\partial}{\partial \varepsilon} \bar{X}(\cdot, \varepsilon)\right|_{\varepsilon=0} \in C_{0}^{2}\left(B \cup I, \mathbb{R}^{3}\right),
$$

$\bar{X}(w, \varepsilon)=X(w)$ for all $\varepsilon \in\left(-\varepsilon_{0}, \varepsilon_{0}\right)$ and all $w \in(B \cup I) \backslash K$ with some compact set $K \subset B \cup I$, and $\left.\bar{X}(\cdot, \varepsilon)\right|_{I}: I \rightarrow \mathscr{S}$ for all $\varepsilon \in\left(-\varepsilon_{0}, \varepsilon_{0}\right)$. $Y$ is called the corresponding variational vector field. Evidently, we deduce

$$
Y(w) \in T_{X(w)} \mathscr{P} \text { for all } y \in I .
$$

Conversely, if $Y \in C_{0}^{2}\left(B \cup I, \mathbb{R}^{3}\right)$ satisfies (2-4), one can show that an admissible variation of the form

$$
\bar{X}(\cdot, \varepsilon)=X+\varepsilon Y+o(\varepsilon)
$$

exists; see [Dierkes et al. 1992, Volume I, p. 333], for example.

We say that $X \in \mathscr{C}(\Gamma, \mathscr{Y})$ is $F$-stationary if the first variation

$$
\delta \mathscr{F}(X, Y):=\left.\frac{d}{d \varepsilon} \mathscr{F}(\bar{X}(\cdot, \varepsilon))\right|_{\varepsilon=0}
$$

vanishes for all admissible variations. An $F$-stationary immersion $X \in \mathscr{C}(\Gamma, \mathscr{Y})$ is called $F$-stable if additionally the second variation

$$
\delta^{2} \mathscr{F}(X, Y)=\left.\frac{d^{2}}{d \varepsilon^{2}} \mathscr{F}(\bar{X}(\cdot, \varepsilon))\right|_{\varepsilon=0}
$$

is nonnegative for all admissible variations. Obviously, any minimizer $X \in \mathscr{C}(\Gamma, \mathscr{S})$ of $\mathscr{F}_{F}$ is $F$-stable, but the converse is not true in general.

Standard computations (see [Clarenz 2002, Section 1] or [Winklmann 2002, Proposition 2.1], for example) show that the first variation for $X \in \mathscr{C}(\Gamma, \mathscr{Y})$ is

$$
\delta \mathscr{F}(X, Y)=-\int_{B} H_{F}\langle Y, N\rangle d A-\int_{I}\left\langle F_{z}(N), X_{u} \wedge Y\right\rangle d u .
$$


Here $H_{F}$ denotes the $F$-mean curvature or anisotropic mean curvature of $X$, defined as follows [Räwer 1993; Clarenz 2002]: Let

$$
N_{F}:=F_{z} \circ N, \quad N_{F}: \bar{B} \backslash\{-1,1\} \rightarrow \mathcal{W}_{F},
$$

describe the generalized Gauss map of $X$ into the Wulff shape. Then $S_{F}:=$ $-d X^{-1} \circ d N_{F}$ is called the $F$-Weingarten operator and

$$
H_{F}:=\operatorname{tr} S_{F} .
$$

For technical reasons, we write

$$
S_{F}:=A_{F} \circ S,
$$

where $S:=-d X^{-1} \circ d N$ denotes the classical Weingarten operator and $A_{F}$ indicates the symmetric, positive-definite endomorphism given by

$$
A_{F}:=d X^{-1} \circ F_{z z}(N) \circ d X .
$$

Note that $A_{F}$ is the identity if $F(z)=|z|$ is the area integrand. Hence, in this case all definitions coincide with the classical notions.

Now assume that $X \in \mathscr{C}(\Gamma, \mathscr{S})$ is $F$-stationary. If we choose $Y=\lambda N$ with $\lambda \in C_{0}^{2}(B, \mathbb{R})$, we infer the identity

$$
H_{F} \equiv 0 \quad \text { on } B
$$

from (2-5) and the fundamental lemma of the calculus of variations. As a consequence,

$$
\int_{I}\left\langle F_{z}(N), X_{u} \wedge Y\right\rangle d u=0 \text { for all } Y \in C_{0}^{2}\left(B \cup I, \mathbb{R}^{3}\right) \text { satisfying (2-4). }
$$

This implies

$$
F_{z}(N(w)) \in T_{X(w)} \mathscr{S} \text { for all } w \in I .
$$

Hence we have the following characterization of $F$-stationary immersions:

Lemma 2.4. Let $F$ be an elliptic Lagrangian and let $\{\Gamma, \mathscr{Y}\}$ be a projectable boundary configuration. $X \in \mathscr{C}(\Gamma, \mathscr{Y})$ is $F$-stationary if and only if $X$ satisfies (2-10) and the contact condition (2-11).

We now derive two general relations, which represent the anisotropic analogues to the well known relations $N_{u}=N \wedge N_{v}, N_{v}=-N \wedge N_{u}$ for conformally parametrized minimal surfaces. These will be particularly important in the derivation of the boundary condition for the normal (Lemma 3.3).

We will use standard shorthands when computing in coordinates, writing indifferently $(u, v)=\left(u^{1}, u^{2}\right)$ and $\varphi_{u}=\varphi_{u^{1}}=\varphi_{, 1}, \varphi_{v}=\varphi_{u^{2}}=\varphi_{, 2}$. We denote the coefficients of the induced metric by $g_{\alpha \beta}=\left\langle X_{, \alpha}, X_{, \beta}\right\rangle$, and the coefficients 
of $\left(g_{\alpha \beta}\right)_{\alpha, \beta=1,2}^{-1}$ by $g^{\alpha \beta}$. Moreover, we abbreviate $g:=\operatorname{det}\left(g_{\alpha \beta}\right)=g_{11} g_{22}-g_{12}^{2}$ with a slight notational overlap. Finally, we let $h_{\alpha \beta}:=g\left(S \partial_{\alpha}, \partial_{\beta}\right)=-\left\langle N_{, \alpha}, X_{, \beta}\right\rangle$ indicate the coefficients of the second fundamental form and $a_{\alpha \beta}=g\left(A_{F} \partial_{\alpha}, \partial_{\beta}\right)=$ $\left\langle F_{z z}(N) X_{, \alpha}, X_{, \beta}\right\rangle$ the coefficients of $g\left(A_{F} \cdot, \cdot\right)$.

As an immediate consequence of (2-7), (2-8), and (2-9) we obtain

$$
\begin{aligned}
S_{F} \partial_{\varepsilon} & =g^{\alpha \beta} a_{\beta \gamma} g^{\gamma \delta} h_{\delta \varepsilon} \partial_{\alpha}, \\
H_{F} & =g^{\alpha \beta} a_{\beta \gamma} g^{\gamma \delta} h_{\delta \alpha},
\end{aligned}
$$

where the Einstein summation convention is in effect. We also need the well known identities

$$
X_{u} \wedge N=-\sqrt{g} g^{2 \alpha} X_{, \alpha}, \quad X_{v} \wedge N=\sqrt{g} g^{1 \alpha} X_{, \alpha}
$$

on $\bar{B} \backslash\{-1,1\}$, valid for an arbitrary immersion $X \in \mathscr{C}(\Gamma, \mathscr{S})$.

Lemma 2.5. For any $F$-stationary immersion $X \in \mathscr{C}(\Gamma, \mathscr{Y})$ we have

$$
\begin{aligned}
& \frac{\partial}{\partial u} F_{z}(N)=\sqrt{g} g^{2 \alpha} a_{\alpha \beta} g^{\beta \gamma} N \wedge N_{, \gamma}, \\
& \frac{\partial}{\partial v} F_{z}(N)=-\sqrt{g} g^{1 \alpha} a_{\alpha \beta} g^{\beta \gamma} N \wedge N_{, \gamma}
\end{aligned}
$$

on $\bar{B} \backslash\{-1,1\}$.

Proof. We prove only the first equality; the argument for the second is similar. First note that both sides of (2-15) are tangential to $X$; more precisely,

$$
\frac{\partial}{\partial u} F_{z}(N)=U^{\alpha} X_{, \alpha}, \quad \sqrt{g} g^{2 \alpha} a_{\alpha \beta} g^{\beta \gamma} N \wedge N_{, \gamma}=V^{\alpha} X_{, \alpha} .
$$

We will show that the coefficients coincide, i.e., $U^{\alpha}=V^{\alpha}$ for $\alpha=1,2$. To this end we use (2-12) obtaining

$$
U^{\alpha}=-g^{\alpha \beta} a_{\beta \gamma} g^{\gamma \delta} h_{\delta 1} .
$$

In order to compute $V^{\alpha}$, we employ (2-14) and deduce

$$
g^{1 \beta}\left\langle N \wedge N_{, \alpha}, X_{, \beta}\right\rangle=\frac{1}{\sqrt{g}} h_{2 \alpha}, \quad g^{2 \beta}\left\langle N \wedge N_{, \alpha}, X_{, \beta}\right\rangle=-\frac{1}{\sqrt{g}} h_{1 \alpha} .
$$

Consequently, we arrive at

$$
V^{1}=g^{2 \beta} a_{\beta \gamma} g^{\gamma \delta} h_{\delta 2}, \quad V^{2}=-g^{2 \beta} a_{\beta \gamma} g^{\gamma \delta} h_{\delta 1} .
$$

Comparison of (2-17) and (2-18) immediately yields $U^{2}=V^{2}$. Furthermore, we see that $V^{1}-U^{1}=g^{\alpha \beta} a_{\beta \gamma} g^{\gamma \delta} h_{\delta \alpha}=H_{F}$, due to (2-13). Because $X$ is supposed to be $F$-stationary, we infer that $U^{1}=V^{1}$. 
Let $\varphi \in C^{2}(B)$ and a smooth vector field $V=V^{\alpha} \partial_{\alpha}$ on $B$ be prescribed. We introduce

$$
\nabla \varphi=g^{\alpha \beta} \varphi_{, \alpha} \partial_{\beta} \quad \text { and } \quad \operatorname{div} V=\frac{1}{\sqrt{g}}\left(\sqrt{g} V^{\alpha}\right)_{, \alpha}=V_{, \alpha}^{\alpha}-\Gamma_{\alpha \beta}^{\alpha} V^{\beta},
$$

the gradient and divergence with respect to $g$; as usual, the $\Gamma_{\alpha \beta}^{\gamma}$ here are the Christoffel symbols, given by the Gauss-Weingarten relations (see [Dierkes et al. 1992, Chapter 1])

$$
X_{, \alpha \beta}=\Gamma_{\alpha \beta}^{\gamma} X_{, \gamma}+h_{\alpha \beta} N .
$$

Following [Clarenz 2002], we define the elliptic operator $\Delta_{F}$ of second order by

$$
\Delta_{F} \varphi:=\operatorname{div}\left(A_{F} \nabla \varphi\right)=\frac{1}{\sqrt{g}}\left(\sqrt{g} g^{\alpha \beta} a_{\beta \gamma} g^{\gamma \delta} \varphi_{, \delta}\right)_{, \alpha} .
$$

We recall the divergence of $A_{F}$, given by the 1-form

$$
\left(\operatorname{div} A_{F}\right)[V]:=V^{\alpha} g^{\beta \gamma} a_{\alpha \beta ; \gamma},
$$

where $a_{\alpha \beta ; \gamma}=a_{\alpha \beta, \gamma}-\Gamma_{\gamma \alpha}^{\delta} a_{\delta \beta}-\Gamma_{\gamma \beta}^{\delta} a_{\alpha \delta}$ denote the coefficients for the covariant derivative of the tensor $g\left(A_{F} \cdot, \cdot\right)$.

The following two identities were established in [Clarenz 2002, Theorem 2; Clarenz and von der Mosel 2004, Corollary 4.3]. Using (2-19) and (2-20), the first of them is derived via the Gauss-Weingarten relations, and the second identity via the Codazzi equation $h_{\alpha \beta ; \gamma}=h_{\beta \gamma ; \alpha}$.

Lemma 2.6. Let $F$ be an elliptic Lagrangian. Then any $F$-stationary immersion $X \in \mathscr{C}(\Gamma, \mathscr{Y})$ fulfills the equations

$$
\begin{aligned}
& \Delta_{F} X-\left(\operatorname{div} A_{F}\right)[\nabla X]=0, \\
& \Delta_{F} N+\operatorname{tr}\left(A_{F} S^{2}\right) N=0
\end{aligned}
$$

on $\bar{B} \backslash\{-1,1\}$.

We conclude this section with a general assumption on $F$, which has two consequences: it forces any $F$-stationary surface $X \in \mathscr{C}(\Gamma, \mathscr{Y})$ in an admissible boundary configuration $\{\Gamma, \mathscr{S}\}$ to map $I$ onto $\Sigma \times \mathbb{R}\left(\right.$ Lemma 3.1), and it ensures that $N^{3}>0$ at the corners (Lemma 3.2).

Condition (W). The Wulff shape $\mathscr{W}_{F}$ meets $E$ perpendicularly, and $\mathscr{W}_{F} \cap E=$ $\partial B_{R}(0) \cap E$ for some radius $R>0$.

According to (2-3), this condition is equivalent to

$$
F_{z}\left(z^{1}, z^{2}, 0\right)=\left(R z^{1}, R z^{2}, 0\right) \text { for all } z \in S^{1} \times\{0\} .
$$




\section{Boundary behaviour of $F$-stationary immersions}

Lemma 3.1. Let $F$ denote an elliptic Lagrangian satisfying Condition (W), and let $\{\Gamma, \mathscr{Y}\}$ represent an admissible boundary configuration. Suppose $X \in \mathscr{C}(\Gamma, \mathscr{Y})$ to be $F$-stationary. Defining $f(w)=\left(X^{1}(w), X^{2}(w)\right): \bar{B} \rightarrow E$, we then infer $f(I)=\Sigma$.

Proof. We follow the proof of [Hildebrandt and Sauvigny 1992, Proposition 1]. Let $s^{*} \in\left(-\infty, s_{1}\right]$ be the largest number such that $f(\bar{B}) \subset H\left(s^{*}\right)$, where we have made the abbreviation

$$
H(s):=\left\{y \in E:\left\langle y-\sigma(s), \sigma^{\prime}(s)\right| \geq 0\right\}, \quad s \in\left(-\infty, s_{1}\right] .
$$

Because $f: \bar{B} \rightarrow E$ is continuous, such an $s^{*}$ exists by (a) in Definition 2.1.

Suppose that $s^{*}<s_{1}$. Then the nonnegative function

$$
\Phi(w):=\left\langle f(w)-\sigma\left(s^{*}\right), \sigma^{\prime}\left(s^{*}\right)\right\rangle, \quad w \in \bar{B},
$$

satisfies the homogeneous elliptic equation $\Delta_{F} \Phi-\left(\operatorname{div} A_{F}\right)[\nabla \Phi]=0$ on $B$, by Lemma 2.6. According to the maximum principle and the choice of $s^{*}$, we can find a point $w_{0} \in \partial B \backslash\{-1,1\}$ with $\Phi\left(w_{0}\right)=0$. From condition (b) in Definition 2.2 and the boundary conditions for $X$ we infer that $w_{0} \in I$ and $f\left(w_{0}\right)=\sigma\left(s^{*}\right)$.

Hopf's boundary point lemma now implies $\Phi_{u}\left(w_{0}\right)=0$ and $\Phi_{v}\left(w_{0}\right)>0$, which we may rewrite as

$$
\left\langle X_{u}\left(w_{0}\right), t\left(X\left(w_{0}\right)\right)\right\rangle=0, \quad\left\langle X_{v}\left(w_{0}\right), t\left(X\left(w_{0}\right)\right)\right\rangle>0 .
$$

Noting that $X_{u}\left(w_{0}\right) \in T_{X\left(w_{0}\right)} \mathscr{S}$ we find $X_{u}\left(w_{0}\right)=X_{u}^{3}\left(w_{0}\right) e_{3}$. This reveals that $N^{3}\left(w_{0}\right)=\left\langle N\left(w_{0}\right), e_{3}\right\rangle=0$, and (2-11) together with Condition (W) imply $N\left(w_{0}\right)=$ $\left\langle N\left(w_{0}\right), t\left(X\left(w_{0}\right)\right)\right\rangle t\left(X\left(w_{0}\right)\right)$. With the aid of (2-14) and (3-1), we now obtain the contradiction

$$
0>-\sqrt{g} g^{2 \alpha}\left\langle X_{, \alpha}\left(w_{0}\right), t\left(X\left(w_{0}\right)\right)\right\rangle=\left\langle X_{u}\left(w_{0}\right) \wedge N\left(w_{0}\right), t\left(X\left(w_{0}\right)\right)\right\rangle=0 .
$$

Thus we conclude $s^{*}=s_{1}$ and hence $f(\bar{B}) \subset H\left(s_{1}\right)$. Similarly, one shows that $f(\bar{B}) \subset H\left(s_{2}\right)$ holds true with

$$
H(s):=\left\{y \in E:\left\langle y-\sigma(s), \sigma^{\prime}(s)\right\rangle \leq 0\right\}, \quad s \in\left[s_{2},+\infty\right) .
$$

A further application of Hopf's boundary point lemma finally yields $f(I)=\Sigma$.

Form Lemma 3.1 we infer that $f(\partial B)=\partial G$. A standard argument then proves transversality to the fixed boundary $C$.

Lemma 3.2. In addition to the assumptions of Lemma 3.1, suppose that $X \in$ $\mathscr{C}(\Gamma, \mathscr{S})$ satisfies Condition $(R)$. Then $N^{3}(w)>0$ for all $w \in C$. 
Proof. By [Clarenz 2002, Theorem 2.3], F-stationary immersions have the convexhull property. Hence, the argument of [Sauvigny 1982, Satz 2] applies and yields the estimate $N^{3}(w)>0$ for arbitrary $w \in C \backslash\{-1,1\}$. See also [Clarenz and von der Mosel 2004, p. 33].

To prove that $N^{3}(w)>0$ for all $w \in C$, suppose that $N^{3}(-1)$ vanished. Then Condition (W), (2-11) and the continuity of $N$ would imply $N(-1) \in T_{P_{1}} \mathscr{S}$; hence

$$
N(-1)=\left\langle N(-1), t\left(P_{1}\right)\right\rangle t\left(P_{1}\right) .
$$

On the other hand, we have $\left\langle N(-1), a\left(P_{1}\right)\right\rangle=0$, where $a\left(P_{1}\right) \in \mathbb{R}^{3}$ denotes a unit tangent vector to $\Gamma$ in $P_{1}$. Combining this with (3-2), we infer the relation

$$
\left\langle t\left(P_{1}\right), a\left(P_{1}\right)\right\rangle=0 .
$$

However, because $\{\Gamma, \mathscr{Y}\}$ is projectable, this is only possible if $\underline{\Gamma}$ meets $\Sigma_{0}$ perpendicularly, in contradiction to condition (a) in Definition 2.2. Thus we must have $N^{3}(-1) \neq 0$, and by continuity even $N^{3}(-1)>0$. The same argument applies to $N^{3}(+1)$ and the proof is complete.

Now we derive a boundary condition for $N^{3}$ on $I$ which generalizes [Hildebrandt and Sauvigny 1995, Proposition 1].

Lemma 3.3. Let $F$ be an elliptic Lagrangian, and let $X \in \mathscr{C}(\Gamma, \mathscr{Y})$ be an $F$ stationary immersion in a projectable boundary configuration $\{\Gamma, \mathscr{Y}\}$. Writing $F=$ $F(N), \kappa=\kappa(X)$, etc., we have

$$
\sqrt{g} g^{2 \alpha} a_{\alpha \beta} g^{\beta \gamma}\left[F^{-1} N^{3}\right]_{, \gamma}=F^{-3} \kappa\left\langle F_{z}, t\right\rangle^{2}\left\langle F_{z} \wedge X_{u}, n\right\rangle N^{3} \quad \text { on } I .
$$

Proof. First note the relation

$$
F\left[F^{-1} N^{3}\right]_{, \gamma}=F\left\langle\left(F^{-1} N\right)_{, \gamma}, e_{3}\right\rangle=-F^{-1}\left\langle F_{z}, N_{, \gamma}\right\rangle\left\langle N, e_{3}\right\rangle+\left\langle N_{, \gamma}, e_{3}\right\rangle .
$$

Because $\left\langle F_{z}(N), N\right\rangle=F(N)$ (by homogeneity), this implies

$$
\left[F^{-1} N^{3}\right]_{, \gamma}=F^{-2}\left\langle N \wedge N_{, \gamma}, F_{z} \wedge e_{3}\right\rangle \quad \text { on } \bar{B} \backslash\{-1,1\} .
$$

In view of Lemma 2.5, we arrive at

$$
\sqrt{g} g^{2 \alpha} a_{\alpha \beta} g^{\beta \gamma}\left[F^{-1} N^{3}\right]_{, \gamma}=F^{-2}\left\langle\frac{\partial}{\partial u} F_{z}, F_{z} \wedge e_{3}\right\rangle=-F^{-2}\left\langle F_{z}, \frac{\partial}{\partial u}\left[F_{z} \wedge e_{3}\right]\right\rangle
$$

on $\bar{B} \backslash\{-1,1\}$.

From (2-11) we conclude $F_{z} \wedge e_{3}=F_{z} \wedge(n \wedge t)=\left\langle F_{z}, t\right\rangle n$ on $I$ and consequently

$$
\left\langle F_{z}, \frac{\partial}{\partial u}\left[F_{z} \wedge e_{3}\right]\right\rangle=\left\langle F_{z}, t\right\rangle\left\langle F_{z}, \frac{\partial}{\partial u} n\right\rangle=\kappa\left\langle F_{z}, t\right\rangle^{2}\left\langle X_{u}, t\right\rangle \quad \text { on } I .
$$

In the last identity, we used the general relation

$$
\langle v, D n(x) w\rangle=\kappa(x)\langle v, t(x)\rangle\langle w, t(x)\rangle \quad \text { for all } v, w \in T_{x} \mathscr{Y}, x \in \mathscr{Y},
$$


due to the cylindrical structure of $\mathscr{Y}$. On the other hand, we compute

$$
\left\langle X_{u}, t\right\rangle F_{z}-\left\langle F_{z}, t\right\rangle X_{u}=\left(X_{u} \wedge F_{z}\right) \wedge\left(e_{3} \wedge n\right)=\left\langle X_{u} \wedge F_{z}, n\right\rangle e_{3} \quad \text { on } I,
$$

by applying (2-11) and $t=e_{3} \wedge n$. By multiplication with $N$ we finally deduce

$$
F\left\langle X_{u}, t\right\rangle=-\left\langle F_{z} \wedge X_{u}, n\right\rangle N^{3} \text { on } I .
$$

Now the relation (3-3) stated above results from (3-4), (3-5), and (3-7).

\section{The second variation}

In this section we consider the second variation of an $F$-stationary immersion $X \in \mathscr{C}(\Gamma, \mathscr{S})$ in a projectable boundary configuration. For second variation formulas under Plateau type boundary conditions we refer the reader to [Räwer 1993], [Fröhlich 2002] and [Clarenz and von der Mosel 2004].

Let $\bar{X}$ be an admissible variation of $X \in \mathscr{C}(\Gamma, \mathscr{Y})$ with the variational vector field $Y \in C_{0}^{2}\left(B \cup I, \mathbb{R}^{3}\right)$. We denote by $N(\varepsilon), d A(\varepsilon), H_{F}(\varepsilon)$ and $Y(\varepsilon)$ geometric quantities evaluated at $\bar{X}(\cdot, \varepsilon)$. Differentiating the first variation formula (2-5), we obtain

$$
\begin{aligned}
& \delta^{2} \mathscr{F}(X, Y) \\
& =\left.\frac{d}{d \varepsilon}(\delta \mathscr{F}(\bar{X}(\cdot, \varepsilon), Y(\varepsilon)))\right|_{\varepsilon=0} \\
& =\left.\frac{d}{d \varepsilon}\left(-\int_{B} H_{F}(\varepsilon)\langle Y(\varepsilon), N(\varepsilon)\rangle d A(\varepsilon)-\int_{I}\left\langle F_{z}(N(\varepsilon)), \bar{X}_{u}(\cdot, \varepsilon) \wedge Y(\varepsilon)\right\rangle d u\right)\right|_{\varepsilon=0} .
\end{aligned}
$$

We now assume that $X$ is $F$-stationary. Then we infer from (2-10) and (2-11) that

$$
\begin{aligned}
\delta^{2} \mathscr{F}(X, Y)=-\int_{B} \frac{\partial}{\partial \varepsilon} & \left.H_{F}(\varepsilon)\right|_{\varepsilon=0}\langle Y, N\rangle d A \\
& \quad-\int_{I}\left\langle\left.\frac{\partial}{\partial \varepsilon} F_{z}(N(\varepsilon))\right|_{\varepsilon=0}, X_{u} \wedge Y\right\rangle d u \\
& \quad-\int_{I}\left\langle F_{z}(N),\left.\frac{\partial}{\partial \varepsilon}\left[\bar{X}_{u}(\cdot, \varepsilon) \wedge Y(\varepsilon)\right]\right|_{\varepsilon=0}\right\rangle d u .
\end{aligned}
$$

According to [2004, Section 4], the variation of the $F$-mean curvature is

$$
\left.\frac{\partial}{\partial \varepsilon} H_{F}(\varepsilon)\right|_{\varepsilon=0}=\Delta_{F} \varphi+\varphi \operatorname{tr}\left(A_{F} S^{2}\right)
$$

where $\varphi=\langle Y, N\rangle$. Integration by parts consequently yields

$$
\begin{aligned}
-\left.\int_{B} \frac{\partial}{\partial \varepsilon} H_{F}(\varepsilon)\right|_{\varepsilon=0}\langle Y, N\rangle d A=\int_{B}\left(g \left(A_{F} \nabla \varphi\right.\right. & \left., \nabla \varphi)-\operatorname{tr}\left(A_{F} S^{2}\right) \varphi^{2}\right) d A \\
& +\int_{I} \varphi \sqrt{g} g^{2 \alpha} a_{\alpha \beta} g^{\beta \gamma} \varphi, \gamma d u
\end{aligned}
$$


Furthermore, we note the relation

$$
\left.\frac{\partial}{\partial \varepsilon} N(\varepsilon)\right|_{\varepsilon=0}=-g^{\alpha \beta}\left\langle Y_{, \beta}, N\right\rangle X_{, \alpha}=g^{\alpha \beta}\left(\left\langle Y, N_{, \beta}\right\rangle-\varphi_{, \beta}\right) X_{, \alpha} .
$$

In view of (2-14) and the identity $F_{z z}(N) N=0$, this gives us

$$
\left\langle\left.\frac{\partial}{\partial \varepsilon} F_{z}(N(\varepsilon))\right|_{\varepsilon=0}, X_{u} \wedge Y\right\rangle=\varphi \sqrt{g} g^{2 \alpha} a_{\alpha \beta} g^{\beta \gamma} \varphi_{, \gamma}-\varphi \sqrt{g} g^{2 \alpha} a_{\alpha \beta} g^{\beta \gamma}\left\langle Y, N_{, \gamma}\right\rangle .
$$

Note that $F_{z z}(N) N=0$ is an immediate consequence of the homogeneity relation (1-1).

Finally, we observe that

$$
\bar{X}_{u}(\cdot, \varepsilon) \wedge Y(\varepsilon)=\left\langle\bar{X}_{u}(\cdot, \varepsilon) \wedge Y(\varepsilon), n(\bar{X}(\cdot, \varepsilon))\right\rangle n(\bar{X}(\cdot, \varepsilon)) \quad \text { on } I,
$$

because $Y(\varepsilon)$ is always tangential to $\mathscr{Y}$. Using $\left\langle F_{z}(N), n\right\rangle=0$ and (3-6), we thus obtain

$$
\left\langle F_{z}(N),\left.\frac{\partial}{\partial \varepsilon}\left[\bar{X}_{u}(\cdot, \varepsilon) \wedge Y(\varepsilon)\right]\right|_{\varepsilon=0}\right\rangle=\kappa\left\langle X_{u} \wedge Y, n\right\rangle\left\langle F_{z}(N), t\right\rangle\langle Y, t\rangle .
$$

Collecting formulas (4-1)-(4-4), we arrive at

$$
\begin{aligned}
\delta^{2} \mathscr{F}(X, Y)= & \int_{B}\left\{g\left(A_{F} \nabla \varphi, \nabla \varphi\right)-\operatorname{tr}\left(A_{F} S^{2}\right) \varphi^{2}\right\} d A \\
& +\int_{I} \varphi \sqrt{g} g^{2 \alpha} a_{\alpha \beta} g^{\beta \gamma}\left\langle Y, N_{, \gamma}\right\rangle d u-\int_{I} \kappa\left\langle X_{u} \wedge Y, n\right\rangle\left\langle F_{z}(N), t\right\rangle\langle Y, t\rangle d u,
\end{aligned}
$$

with $\varphi=\langle Y, N\rangle$.

Variational vector fields of the form $Y=\lambda F(N)^{-1} F_{z}(N)$ with some function $\lambda \in C_{0}^{2}(B \cup I, \mathbb{R})$ are of special interest. According to $\langle Y, N\rangle=\lambda$ and $\left\langle Y, N_{, \gamma}\right\rangle=$ $\lambda F(N)^{-1} F(N)_{, \gamma}$, we obtain:

Theorem 4.1. Let $F$ be an elliptic Lagrangian and let $X \in \mathscr{C}(\Gamma, \mathscr{S})$ be an $F$ stationary immersion in a projectable boundary configuration $\{\Gamma, \mathscr{Y}\}$. For any $\lambda \in$ $C_{0}^{2}(B \cup I, \mathbb{R})$ the second variation of $X$ in the direction $Y=\lambda F(N)^{-1} F_{z}(N)$ is then given by

$$
\begin{aligned}
\delta^{2} \mathscr{F}(X, \lambda)=\int_{B} & \left(g\left(A_{F} \nabla \lambda, \nabla \lambda\right)-\operatorname{tr}\left(A_{F} S^{2}\right) \lambda^{2}\right) d A \\
& +\int_{I} \lambda^{2} \sqrt{g} F(N)^{-1} g^{2 \alpha} a_{\alpha \beta} g^{\beta \gamma} F(N)_{, \gamma} d u \\
& \quad-\int_{I} \lambda^{2} \kappa F(N)^{-2}\left\langle X_{u} \wedge F_{z}(N), n(X)\right\rangle\left\langle F_{z}(N), t(X)\right\rangle^{2} d u
\end{aligned}
$$


Here we have set $\delta^{2} \mathscr{F}(X, \lambda):=\delta^{2} \mathscr{F}\left(X, \lambda F(N)^{-1} F_{z}(N)\right)$. In particular, for all $F$-stable $X$ we have

$$
\delta^{2} \mathscr{F}(X, \lambda) \geq 0
$$

with arbitrary $\lambda \in C_{0}^{2}(B \cup I, \mathbb{R})$.

Remark. Observe that (4-6) remains true for $\lambda \in H_{2}^{1}(B, X) \cap C_{0}^{0}(B \cup I)$, where we have set

$$
H_{2}^{1}(B, X)=\left\{\lambda: B \rightarrow \mathbb{R} \text { measurable }: \int_{B}\left\{\lambda^{2}+|\nabla \lambda|^{2}\right\} d A<+\infty\right\} .
$$

Using the essential assumption (W) and the regularity hypothesis (R), we now show that $\left(N^{3}\right)^{-}:=\min \left\{N^{3}, 0\right\}$ is an admissible test function.

Lemma 4.2. Let $F$ be an elliptic Lagrangian satisfying the Condition (W), and let $\{\Gamma, \mathscr{Y}\}$ be an admissible boundary configuration. Suppose furthermore that $X \in \mathscr{C}(\Gamma, \mathscr{S})$ denotes an $F$-stationary immersion satisfying Condition $(R)$. Then $\left(N^{3}\right)^{-}$lies in $H_{2}^{1}(B, X) \cap C_{0}^{0}(B \cup I)$.

Proof. Clearly, $\left(N^{3}\right)^{-} \in C_{0}^{0}(B \cup I)$ in view of the continuity assumption (2-2) and Lemma 3.2.

In order to prove $\left(N^{3}\right)^{-} \in H_{2}^{1}(B, X)$, we utilize (2-1) and argue as follows: Fix $w \in B$, and let $\left\{e_{1}, e_{2}\right\}$ be an orthonormal basis of $T_{w} B$ such that $S e_{i}=\kappa_{i} e_{i}$ for $i=1,2$ hold true at this point; here $\kappa_{1}, \kappa_{2}$ denote the principal curvatures of $X$. Because $H_{F}$ vanishes, we have the relation

$$
\alpha_{1} \kappa_{1}+\alpha_{2} \kappa_{2}=0
$$

with $\alpha_{i}:=g\left(A_{F} e_{i}, e_{i}\right)$. Now we estimate $0<\Lambda^{-} \leq \alpha_{1}, \alpha_{2} \leq \Lambda^{+}<\infty$ where

$$
\Lambda^{-}:=\inf _{\substack{z \in S^{2} \\ \zeta \in z^{\perp} \backslash\{0\}}} \frac{\left\langle F_{z z}(z) \zeta, \zeta\right\rangle}{|\zeta|^{2}}, \quad \Lambda^{+}:=\sup _{\substack{z \in S^{2} \\ \zeta \in z^{\perp} \backslash\{0\}}} \frac{\left\langle F_{z z}(z) \zeta, \zeta\right\rangle}{|\zeta|^{2}}
$$

give a lower and upper bound for the eigenvalues of $A_{F}$, respectively. A combination with (4-7) yields the estimate

$$
\kappa_{1}^{2}+\kappa_{2}^{2} \leq 2\left(\frac{\Lambda^{+}}{\Lambda^{-}}\right)|K|
$$

where $K=\kappa_{1} \kappa_{2}$ denotes the Gaussian curvature of $X$. Due to $|\nabla N|^{2}=\kappa_{1}^{2}+\kappa_{2}^{2}$, we conclude that

$$
\int_{B}|\nabla N|^{2} d A \leq 2\left(\frac{\Lambda^{+}}{\Lambda^{-}}\right) \int_{B}|K| d A .
$$

Thus $N$ lies in $H_{2}^{1}(B, X)$ and the assertion follows. 
Remark. Sauvigny [1990, Lemma 7] has used similar arguments in order to establish curvature estimates for immersions of minimal-surface type in weighted conformal parameters.

\section{Projectability and uniqueness}

In this section we prove our main results.

Theorem 5.1. Let $F$ denote an elliptic Lagrangian satisfying Condition (W), and let $\{\Gamma, \mathscr{Y}\}$ constitute an admissible boundary configuration. Furthermore, let $X \in$ $\mathscr{b}(\Gamma, \mathscr{Y})$ describe an $F$-stable immersion satisfying Condition $(R)$. Then we have

$$
N^{3}(w)>0 \quad \text { on } \bar{B}
$$

and $X$ can be represented as a graph over the $x^{1}, x^{2}$-plane, i.e., we have the parametrization $x^{3}=\zeta\left(x^{1}, x^{2}\right),\left(x^{1}, x^{2}\right) \in \bar{G}$, with some function

$$
\zeta \in C^{3}\left(\bar{G} \backslash\left\{p_{1}, p_{2}\right\}\right) \cap C^{0}(\bar{G}),
$$

where $p_{1}:=\sigma\left(s_{1}\right)$ and $p_{2}:=\sigma\left(s_{2}\right)$.

Proof. According to Lemma 4.2 and the preceding remark, we know that the function $\omega^{-}:=\min \{\omega, 0\} \in H_{2}^{1}(B, X) \cap C_{0}^{0}(B \cup I)$ with $\omega:=N^{3}$ is admissible in the second variation formula (4-5). Using (2-22), an integration by parts yields

$$
\begin{aligned}
\delta^{2} \mathscr{F}\left(X, \omega^{-}\right)=- & \int_{I} \omega^{-} \sqrt{g} g^{2 \alpha} a_{\alpha \beta} g^{\beta \gamma} N_{, \gamma}^{3} d u \\
& \quad+\int_{I} \omega^{-} N^{3} \sqrt{g} F(N)^{-1} g^{2 \alpha} a_{\alpha \beta} g^{\beta \gamma} F(N)_{, \gamma} d u \\
& \quad-\int_{I} \omega^{-} N^{3} \kappa F(N)^{-2}\left\langle X_{u} \wedge F_{z}(N), n(X)\right\rangle\left\langle F_{z}(N), t(X)\right\rangle^{2} d u \\
=- & \int_{I} \omega^{-} \sqrt{g} F(N) g^{2 \alpha} a_{\alpha \beta} g^{\beta \gamma}\left[F(N)^{-1} N^{3}\right], \gamma d u \\
& \quad-\int_{I} \omega^{-} N^{3} \kappa F(N)^{-2}\left\langle X_{u} \wedge F_{z}(N), n(X)\right\rangle\left\langle F_{z}(N), t(X)\right\rangle^{2} d u .
\end{aligned}
$$

Lemma 3.3 then reveals that $\delta^{2} \mathscr{F}\left(X, \omega^{-}\right)=0$.

From here onwards we can argue as in [Hildebrandt and Sauvigny 1995]: Defining $\Phi(\varepsilon):=\delta^{2} \mathscr{F}\left(X, \omega^{-}+\varepsilon \varphi\right)$ with arbitrary $\varphi \in C_{0}^{\infty}(B)$, the stability inequality (4-6) implies $\Phi^{\prime}(0)=0$. This is equivalent to

$$
\int_{B}\left(g\left(A_{F} \nabla \omega^{-}, \nabla \varphi\right)-\operatorname{tr}\left(A_{F} S^{2}\right) \omega^{-} \varphi\right) d A=0 \quad \text { for all } \varphi \in C_{0}^{\infty}(B) .
$$


Because $\omega^{-}$lies in $C_{0}^{0}(B \cup I)$, Moser's weak Harnack inequality yields $\omega^{-} \equiv 0$, i.e., $N^{3} \geq 0$ on $\bar{B}$. This gives us $N^{3}>0$ on $B \cup C$, which is a consequence of (2-22) and $N^{3}>0$ near $C$ in conjunction with Harnack's inequality.

Finally, we establish that $N^{3}>0$ on $I$. Indeed, if $N^{3}\left(w_{0}\right)=0$ were true for some $w_{0} \in I$, Lemma 3.3 would imply

$$
g^{2 \alpha} a_{\alpha \beta} g^{\beta \gamma} N_{, \gamma}^{3}\left(w_{0}\right)=0 .
$$

On the other hand, Hopf's boundary point lemma yields $N_{u}^{3}\left(w_{0}\right)=0$ and $N_{v}^{3}\left(w_{0}\right) \neq$ 0 . According to the definiteness of the matrix $\left(g^{\delta \alpha} a_{\alpha \beta} g^{\beta \gamma}\right)$ we have $g^{2 \alpha} a_{\alpha \beta} g^{\beta 2} \neq 0$, and (5-2) generates a contradiction proving (5-1).

Due to Lemma 3.1, we have $\left.f\right|_{\partial B}: \partial B \rightarrow \partial G$ topologically. Indeed, we already know $\left.f\right|_{C}: C \rightarrow \underline{\Gamma}$ topologically by assumption, and (5-1) yields $J_{f}(w)>0$ on $\bar{B} \backslash$ $\{-1,1\}$, thus $\left|f_{u}\right|>0$ on $I$ as well. In addition, an index argument yields $\left.f\right|_{B}: B \rightarrow$ $G$ topologically. In fact, this follows from $J_{f}(w)>0$ on $B$, the boundary behaviour of $f$ and the well known index-sum formula, compare [Sauvigny 2006, Chapter III]. Finally, the implicit function theorem reveals $\zeta\left(x^{1}, x^{2}\right):=X^{3} \circ f^{-1}\left(x^{1}, x^{2}\right) \in$ $C^{3}\left(\bar{G} \backslash\left\{p_{1}, p_{2}\right\}\right) \cap C^{0}(\bar{G})$.

We conclude with a geometric uniqueness result.

Theorem 5.2. Let $F$ be an elliptic Lagrangian satisfying Condition (W), and let $\{\Gamma, \mathscr{Y}\}$ denote an admissible boundary configuration. Then, apart from reparametrizations, there exists at most one $F$-stable immersion $X \in \mathscr{C}(\Gamma, \mathscr{Y})$ satisfying Condition (R).

Remark. Again we refer the reader to [Hildebrandt and Sauvigny 1995] and [Müller 2005], concerning comparable results for stable minimal surfaces and surfaces of prescribed mean curvature, respectively. The existence of $F$-stationary immersions with partially free boundaries has not yet been proved, but see [Hildebrandt and von der Mosel 2002] for related results. For the construction of an embedded $F$-minimal surface bounding a closed smooth Jordan curve $\Gamma \subset \mathbb{R}^{3}$, see [White 1991].

Proof of Theorem 5.2. According to Theorem 5.1, any $F$-stable immersion $X \in$ $\mathscr{C}(\Gamma, \mathscr{S})$ satisfying Condition $(\mathrm{R})$ can be represented as a graph

$$
\zeta\left(x^{1}, x^{2}\right)=X^{3} \circ f^{-1}\left(x^{1}, x^{2}\right) \in C^{3}\left(\bar{G} \backslash\left\{p_{1}, p_{2}\right\}\right) \cap C^{0}(\bar{G}) .
$$

Moreover, this graph representation has the same orientation as $X$, due to (5-1). Because $X$ is $F$-stationary, the height function $\zeta$ is a critical point of the nonparametric functional

$$
\mathfrak{F}[\zeta]=\int_{G} f(D \zeta) d x
$$


where we have written $f(q)=f\left(q_{1}, q_{2}\right):=F\left(-q_{1},-q_{2}, 1\right) \in C^{3}\left(\mathbb{R}^{2}\right)$ and $D \zeta=$ $\left(\zeta_{x^{1}}, \zeta_{x^{2}}\right)$. In particular, the function $\zeta$ solves the mixed boundary value problem

$$
\begin{gathered}
Q \zeta:=\frac{\partial}{\partial x^{\alpha}}\left(\frac{\partial f}{\partial q_{\alpha}}(D \zeta)\right)=0 \quad \text { on } G, \\
\zeta=\gamma \quad \text { on } \underline{\Gamma}, \quad\left\langle f_{q}(D \zeta), \nu\right\rangle=0 \quad \text { on } \Sigma .
\end{gathered}
$$

Here $\gamma=\gamma\left(x^{1}, x^{2}\right)$ denotes the given height function above $\underline{\Gamma}$, and $v=v\left(x^{1}, x^{2}\right)$ is the normal of $\Sigma$ which points to the exterior of $G$. In view of the ellipticity of $F$, we infer the minimal surface type condition

$$
\frac{\partial^{2} f}{\partial q_{\alpha} \partial q_{\beta}}(q) \xi^{\alpha} \xi^{\beta} \geq \frac{\Lambda^{-}}{\sqrt{1+|q|^{2}}}\left(|\xi|^{2}-\frac{\langle q, \xi\rangle^{2}}{1+|q|^{2}}\right)
$$

for all $q=\left(q_{1}, q_{2}\right), \xi=\left(\xi^{1}, \xi^{2}\right) \in \mathbb{R}^{2}$, where $\Lambda^{-}$is the positive number given by (4-8); see also [Finn 1954; Simon 1977].

Now assume we had two $F$-stable immersions $X_{1}, X_{2} \in \mathscr{C}(\Gamma, \mathscr{Y})$ such that $\zeta_{l}=X_{l}^{3} \circ f_{l}^{-1}\left(x^{1}, x^{2}\right) \in C^{3}\left(\bar{G} \backslash\left\{p_{1}, p_{2}\right\}\right) \cap C^{0}(\bar{G})$ with $l=1,2$ satisfy (5-3). Then the difference function $\zeta:=\zeta_{1}-\zeta_{2}$ solves a linear elliptic equation on $G$ with their coefficients in $C^{1}\left(\bar{G} \backslash\left\{p_{1}, p_{2}\right\}\right)$, compare [Gilbarg and Trudinger 1983] and [Sauvigny 2006, Chapter VI, §2]. According to the maximum principle, the function $\zeta$ has to assume its maximum and minimum on $\Sigma \cup \underline{\Gamma}$.

We now infer

$$
\langle M \cdot D \zeta, v\rangle=0 \quad \text { on } \Sigma
$$

from the second boundary condition in (5-3), where we abbreviated

$$
M=M\left(x^{1}, x^{2}\right):=\int_{0}^{1} f_{q q}\left(t D \zeta_{1}+(1-t) D \zeta_{2}\right) d t .
$$

If the function $\zeta$ assumed a positive maximum at the point $x_{0} \in \Sigma$, Hopf's boundary point lemma would imply $D \zeta\left(x_{0}\right)=\left\langle D \zeta\left(x_{0}\right), v\right\rangle v$ with $\left\langle D \zeta\left(x_{0}\right), v\right\rangle>0$. In view of (5-4), we would have

$$
\left\langle M \cdot D \zeta\left(x_{0}\right), v\right\rangle=\left\langle D \zeta\left(x_{0}\right), v\right\rangle\langle M v, v\rangle>0,
$$

contradicting (5-5). Similarly, one excludes a negative minimum on $\Sigma$. Hence we infer $\zeta \equiv 0$ on $\bar{G}$ and the announced result follows.

\section{References}

[Clarenz 2002] U. Clarenz, "Enclosure theorems for extremals of elliptic parametric functionals", Calc. Var. Partial Diff. Eq. 15:3 (2002), 313-324. MR 2003m:49003 Zbl 1018.53006

[Clarenz and von der Mosel 2004] U. Clarenz and H. von der Mosel, "On surfaces of prescribed F-mean curvature”, Pacific J. Math. 213:1 (2004), 15-36. MR 2005b:53010 Zbl 1058.53003 
[Dierkes et al. 1992] U. Dierkes, S. Hildebrandt, A. Küster, and O. Wohlrab, Minimal surfaces (2 vol.), Grundlehren der Math. Wissenschaften 295-296, Springer, Berlin, 1992. MR 94c:49001b Zbl 0777.53012

[Finn 1954] R. Finn, “On equations of minimal surface type”, Ann. of Math. (2) 60 (1954), 397-416. MR 16,592b Zbl 0058.32501

[Fröhlich 2002] S. Fröhlich, "Curvature estimates for $\mu$-stable $G$-minimal surfaces and theorems of Bernstein type", Analysis (Munich) 22:2 (2002), 109-130. MR 2003f:35108 Zbl 01803957

[Gilbarg and Trudinger 1983] D. Gilbarg and N. S. Trudinger, Elliptic partial differential equations of second order, 2nd ed., Grundlehren der Math. Wissenschaften 224, Springer, Berlin, 1983. MR 86c:35035 Zbl 0562.35001

[Hildebrandt and Sauvigny 1991] S. Hildebrandt and F. Sauvigny, "Embeddedness and uniqueness of minimal surfaces solving a partially free boundary value problem", J. Reine Angew. Math. 422 (1991), 69-89. MR 92k:58056 Zbl 0729.53014

[Hildebrandt and Sauvigny 1992] S. Hildebrandt and F. Sauvigny, "On one-to-one harmonic mappings and minimal surfaces”, Nachrichten Akad. Wiss. Göttingen Math.-Phys. Kl. II 3 (1992), 21. MR 94b:49069 Zbl 0794.35050

[Hildebrandt and Sauvigny 1995] S. Hildebrandt and F. Sauvigny, "Uniqueness of stable minimal surfaces with partially free boundaries”, J. Math. Soc. Japan 47:3 (1995), 423-440. MR 96a:58061 Zbl 0831.53006

[Hildebrandt and von der Mosel 1999] S. Hildebrandt and H. von der Mosel, "On two-dimensional parametric variational problems", Calc. Var. Partial Diff. Eq. 9 (1999), 249-267. MR 2000h:49006 Zbl 0934.49022

[Hildebrandt and von der Mosel 2002] S. Hildebrandt and H. von der Mosel, "The partially free boundary problem for parametric double integrals", pp. 145-165 in Nonlinear problems in mathematical physics and related topics, vol. I, edited by M. S. Birman et al., Kluwer/Plenum, New York, 2002. MR 2004b:49004 Zbl 1055.49024

[Hildebrandt and von der Mosel 2005] S. Hildebrandt and H. von der Mosel, "Conformal representation of surfaces, and Plateau's problem for Cartan functionals", Preprint 2005-07-05, RWTH, Aachen, 2005. To appear in Riv. Mat. Univ. Parma.

[Kneser 1926] H. Kneser, “Lösung der Aufgabe 41”, Jahresber. Deutsch. Math.-Ver. 35 (1926), 123124.

[Koiso and Palmer 2006] M. Koiso and B. Palmer, "Stability of anisotropic capillary surfaces between two parallel planes", Calc. Var. Partial Diff. Eq. 25:3 (2006), 275-298. MR 2006j:58018 Zbl 05014072

[Müller 2005] F. Müller, "On stable surfaces of prescribed mean curvature with partially free boundaries”, Calc. Var. Partial Diff. Eq. 24:3 (2005), 289-308. MR 2007c:53013 Zbl 1079.53018

[Radó 1926] T. Radó, “Aufgabe 41”, Jahresber. Deutsch. Math.-Ver. 35 (1926), 49.

[Räwer 1993] K. Räwer, Stabile Extremalen parametrischer Doppelintegrale im $\mathbb{R}^{3}$, Dissertation, Universität Bonn, 1993.

[Sauvigny 1982] F. Sauvigny, "Flächen vorgeschriebener mittlerer Krümmung mit eineindeutiger Projektion auf eine Ebene”, Math. Z. 180:1 (1982), 41-67. MR 83j:53004 Zbl 0465.53003

[Sauvigny 1990] F. Sauvigny, "Curvature estimates for immersions of minimal surface type via uniformization and theorems of Bernstein type", Manuscripta Math. 67:1 (1990), 69-97. MR 91b: 53010 Zbl 0703.53050 
[Sauvigny 2006] F. Sauvigny, Partial differential equations, 1: Foundations and integral representations, Universitext, Springer, Berlin, 2006. Expanded translation of Partielle Differentialgleichungen der Geometrie und der Physik: Grundlagen und Integraldarstellungen, Springer, Berlin, 2004. MR 2007c:35001 Zbl 1049.35001

[Simon 1977] L. Simon, "Equations of mean curvature type in 2 independent variables", Pacific J. Math. 69:1 (1977), 245-268. MR 56 \#13099 Zbl 0354.35040

[Taylor 1978] J. E. Taylor, “Crystalline variational problems", Bull. Amer. Math. Soc. 84:4 (1978), 568-588. MR 58 \#12649 Zbl 0392.49022

[White 1991] B. White, "Existence of smooth embedded surfaces of prescribed genus that minimize parametric even elliptic functionals on 3-manifolds", J. Differential Geom. 33:2 (1991), 413-443. MR 92e:58048 Zbl 0737.53009

[Winklmann 2002] S. Winklmann, "Isoperimetric inequalites involving generalized mean curvature”, Analysis (Munich) 22:4 (2002), 393-403. MR 2003j:53096 Zbl 1033.53052

[Winklmann 2003] S. Winklmann, "Existence and uniqueness of F-minimal surfaces", Ann. Global Anal. Geom. 24:3 (2003), 269-277. MR 2004g:53015 Zbl 1027.53068

Received August 25, 2005.

FRANK MÜLLER

BRANDENBURGISCHE TECHNISCHE UNIVERSITÄT COTTBUS

INSTITUT FÜR MATHEMATIK

KONRAD-ZuSE-STRASSE 1

03044 COTTBUS

GERMANY

mueller@math.tu-cottbus.de

SVEN WINKLMANN

UNIVERSITÄT DUISBURG-ESSEN

CAMPUS DUISBURG

FACHBEREICH MATHEMATIK

47048 DUISBURG

GERMANY

winklmann@math.uni-duisburg.de

http://www.uni-duisburg.de/FB11/DGL/Winklmann/wink 